\title{
IS A NON-LEAKING FILLING NECESSARY TO TOOTH SAL- VATION WHEN AMALGAM IS USED?
}

\author{
By Wm. E. Harper, D. D. S., Chicago, III.
}

(Read before the Illinois State Dental Society, Peoria, May 13-16, 1919.)

\section{$\mathrm{I}^{\mathrm{N}}$} N PRESENTING this question, I wish to make one statement of fact for your guidance, namely: That more than ninety per cent of the amalgam fillings made for me for test purposes leaked as soon as subjected to air pressure from within; and more than seventy-five per cent were so defective that the imperfections within the cavity were apparent to the naked eye when the cavity walls were removed from the filling for observation.

Notwithstanding these deplorable results in adaptation, and other common defects resulting from faulty amalgam technic, clinical observation shows conclusively that amalgam fillings generally save the teeth, and with the teeth our reputations.

This fact explains why it has been possible for us to continue its use, which would not be possible with any other filling material at present known.

With the skill and knowledge developed by twenty years experience in testing alloys, and provided with such facilities for measuring results as were known at the date of my last report to this Society, my best average in test fillings was about twenty per cent that would resist four or more pounds air pressure; and of this twenty per cent more than half would leak under three pounds within nine months after the time they were made, thus showing a lack of stability.

The character of these common faults in adaptation, and other defects, and the wide variation in the quality of our amalgam work, ranging from disgraceful to par excellence, convinced me that the fault was largely in our amalgam procedure and this conviction decided my line of investigation.

This paper may be considered as a final report to that made to this Society and published in the 1912 proceedings. At that time the result of four years effort to improve our amalgam work was most discouraging on account of the perplexing inconsistencies of results attained.

Since that time I have continued my work, determined to eliminate these faults in adaptation if it were possible. Today I come prepared to prove the cause of our amalgam failures and to demonstrate that, with a proper amalgam technic and a dependable high grade alloy, eighty-five to ninety-five per cent of our amalgam fillings may be made sufficiently perfect in their adaptation to resist leakage at three to thirty pounds air pressure under practical operating conditions, with greatly increased resistance to crushing stress and flow, and with approximately uniform results.

Under continued observation during the past five years my test fillings are showing stability by continuing to stand up to their original test in simple round and proximo-occlusal test cavities.

A brief statement of my progress in this work would seem interesting and may enlighten you on the relative importance of the several modifications in our amalgam technic presented as essential to results. 
Within a short time following our annual meeting of 1912, I discovered, more or less accidentally, the importance of a decided plasticity of the amalgam mass during the packing. This condition of plasticity, in which little or no crepitus can be observed during the filling of the greater part of the cavity, is accomplished by retaining the excess mercury used to make the mix: to be removed by forcible condensation during the packing instead of expressing it during the final kneading or mixing.

With this increased plasticity I jumped from about twenty to seventy per cent of fillings sufficiently perfect to resist air pressure of three to thirty pounds.

Encouraged by this great improvement, further effort soon led to a recognition of deficiencies and lack of uniformity in the application of condensing force.

It required a very little experience with an orderly packing to recognize that fcrce not uniformly applied, was largely the explanation of the lack of uniformity in adaptation, strength, and stability. This conclusion led to the adoption of an orderly stepping of the plugger, by which means my average was increased from seventy to eighty per cent. Further effort to attain uniformity resulted in the procedure described as tamping and stepping the cavity walls, operative details that show an increase of ten to fifteen per cent in adaptation.

With these modifications in amalgam procedure I am able to make approximately ninety per cent perfect fillings under the air test, instead of less than ten per cent stable fillings when I undertook the investigation of our amalgam procedure.

The importance of a decided plasticity and uniform or orderly condensation was presented to the profession for the first time I believe on the occasion of the Golden Anniversary of our Society, held in Chicago in 1914. Since that time I have been the beneficiary of a few friendly slams and jokes on what is described as a sloppy mix. Today, experience has made me more insistant than ever on the importance of this procerdure, confidant that it cannot be disproved by practical demonstration. In this connection, I hold myself prepared and happy to enter into any competition with the best amalgam operators my profession can provide as the best means to prove or disprove the worth of this or any other detail of the standardized amalgam technic presented as the result of this effort.

The first intimation $I$ received of the generally defective adaptation in our amalgam work came about twelve years ago with the installation of the air pressure test as a measure of adaptation instead of the amalgam micrometer. With this knowledge, it was easy to account for the dicsouraging tooth discoloration, the one heretofore insurmountable objection to the more general use of amalgam.

This knowledge caused me to confine mv effort to this phase of the work, a practical amalgam technic, for which the combined experience of an every day practice, teacher of operative technic and operative dentistry, and student of amalgam, might better fit me to develop.

After progressing in the work to the pcint of recognition of the necessity of plasticity and uniform condensation, I soon learned by experience in my tests, that adaptation, resistance to crushing stress and flow, and stability, are one and all dependant upon the same details of operative procedure.

The market provides dependable high grade alloys, but the development of the important qualities of adaptation, strergth, stability and proper form and finish, are entirely a question of our operative skill; and the need for perfect results is urgent and within the reach of every practitioner. The one detail of amalgam procedure most necessary to results is the one most difficult to attain. Namely: Proper condensation. The importance of this detail must be demon- 
strated by practical laboratory work in the technical courses of our Dental Schools, and the foundation must be correctly and securely laid for its fullest development by later experience, otherwise it is seldom acquired.

I would answer my question in the title of this paper by saying, Yes, because tooth discoloration is always the result of a leaking filling. This heretofore insurmountable and legitimate objection to its proper and more extended use can be removed by a proper amalgam technic, with the possibility of assured stability, the great tooth preserving attribute of amalgam, its strength and resistance to attrition, low cost, and comparative ease of manipulation, whatever the condition or wherever the position of the cavity, demands a square deal.

In my contributions on this subject made during the past five years, I have formulated and insisted upon the following steps, in the order presented, as a standardized amalgam technic, essential to adaptation, strength, and stability.

\section{PROPORTIONS.}

Nieed not be exact but mercury should be plentiful, sufficient to avoid any trace of dryness in the mixing and just within the other extreme of liquid plasticity.

Either extreme is unfavorable to thoro amalgamation.

When the proportions have been decided upon by a trial mix, it will save time and material to use the small amalgam balance sold for that purpose.

The amount of mercury used in making a mix has nothing whatever to do with the excess mercury in the finished filling.

\section{Mixing.}

All high grade alloys amalgamate rather slowly. It requires thoro rubbing of the particles in the presence of mercury to develop thoro amalgamation, conditions that prohibit a hand mix. Mixing is best accomplished by rapid rubbing in a deep mortar for a minimum time of three minutes. A measure of time is the only dependable guide to a thoro mix.

Thoro mixing is necessary to attain that plasticity most favorable to adaptation. It is also necessary to the greatest strength of the filling, and it is the means by which we may practically eliminate that movement immediately following the placing of the filling. This early movement is common to all alloys.

Mercury may be added or expressed during the mixing to meet the requirements stated.

\section{Plastictty.}

In all average large and proximoocclusal cavities the amalgam mass must be extremely plastic during the filling of the first two-thirds of the cavity to insure adaptation in the use of high grade alloys.

This important requirement is best accomplished by retaining sufficient of the excess mercury (commonly removed during the final mixing or kneading) to be removed after it has served its purpose, by thoro, forceful, and orderly packing.

The excess mercury generally removed during the final mixing is not dangerous to the stability of the filling because this mercury is a surplus always removed by verv ordinary packing force.

The excess mercury that requires our special attention is the final excess; the complete removal of this dangerous excess is absolutely and solely dependent upon thoro, forceful, and orderly condensation.

Decided plasticity and the importance of uniform condensation are two operative details, not previously recognized by the profession as essential to results, that must be accepted if we would make, with reasonable uniformity, non-leaking and stable amalgam fillings.

\section{TAMPING.}

Was designed and has proved to be an aid to uniform condensation. Tamping is done by lightly tapping each piece of amalgam added with the plugger until it spreads as a uniformly thick, dense, and plastic layer in contact with the 
surrounding walls, and well into the angles, then we may proceed to condense. This procedure provides uniform bulk and plasticity under each thrust of the plugger. Experience shows a material improvement in adaptation when this detail is carried out.

\section{PACKING.}

The packing should be as forcible and thoro as possible and applied with the greatest possible uniformity. The most practical means of accomplishing this in the mouth is by an orderly stepping of the plugger.

Following the detail of tamping, we start the packing of each piece of amalgam with a medium or large plugger at the center of the filling, stepping a little less than its diameter in one direction in a gradually enlarging circle until the cavity walls are reached. The final condessation of each piece of amalgam is done by stepping the cavity walls.

To step the cavity walls we use the smallest plugger (size about twelve by eighteen tenths of a millimeter in diameter) stepping a little less than its long diameter immediately against the walls and well into the angles once around the cavity.

With an orderly packing, the excess mercury is always driven in one direction, outward, where it is at all times free to come to the surface to be removed as it appears.

Packing without order results in irregular condensation. Those parts of the filling thoroly condensed will contain no excess mercury, and, as a result will he abundantly strong, the parts of the filling less thoroly condensed will contain excess mercury, and, as a result the filling will be weak and unstable with corresponding possible defects in adaptation.

With an orderly packing the final excess mercury finds its last lodgment immediately against the cavity walls and particularly in the angles; at which points it will exert its worst effects if allowed to remain. Forcibly stepping the cavity walls is the one procedure that will most certainly remove this dangerous excess and at the same time perfect the adaptation.

In irregular cavities orderly stepping mist be carried out as far as it is practicable.

The operating details of condensation ar: the most exacting and difficult to icquire because they must be carried out simultaneously. To develop the skill necessary to uniform results, demands our most thoughtful consideration until correct habits have been formed.

As the filling approaches completion, the amalgam should be gradually less plastic, until at the finish, the excess murcury may be pinched out forcibly to the point where it still remains a workable mass.

The cavity should be filled with an excess, covering well all margins, after which the surface should be thoroly condensed with the largest plugger. This final condensation may be done with pluggers sufficiently large to cover the whole fllling, in combination with the heaviest possible mallet force; or the entire filling should be forcibly compressec with the thumb, holding the amalgam against all margins at the same time for a few moments. This will express the final excess mercury.

The filling should now remain undisturbed for about three minutes after which, it should be trimmed and carved to correct proximal and occlusal form, as far as this is possible; and the surface may be made smooth by light burnishing, and the use of soft brush wheels and pumice. To be given the final polish at a subsequent sitting.

\section{The Matrix.}

The heavy packing force required to insure the complete removal of excess mercury, makes the use of the matrix imperative in all cavities in which one or more of the surrounding walls are missing. 
The matrix may be held by ligature, or by use of one of the mechanical retainers sold for that purpose. It should b*: held securely and should be reasonably well adapted to the tooth at all peints along the cavity margins.

It is very much easier to avoid a large bulk of amalgam in the interproximate space and overlap at the gingival margin than it is to trim it off during or after the setting of the amalgam.

For this purpose, I insert a round tapering wood toothpick from the lingual to wedge the matrix solidly against the gingival margin. This wedge will assist materially in preserving the interproximate space and will save much time in trimming the filling to form.

The matrix must be so held that it may be adjusted, or stretched, to restore the contact point when building the filling; and, wherever necessary and possible, sufficient separation should be made to secure enough room in the interproximate space, to restore its function.

The importance of restoring the interproximate space, contacts, tooth form, and the polishing of an amalgam filling, has not been overlooked in the preparation of this paper. The technic involved in this phase of amalgam work requires much skill, and is of such importance and extent as to justify separate consideration by those better qualified to teach it. To those who seek such help, I know of no better authority than Dr. J. M. Prime, of Omaha, Neb., who's contributions to our literature on this subject will be found to meet the most exacting requirements.

The merits of these details of procedure and the conclusions I present may be best judged when considered in conjunction with the investigations presented in a paper written by ArthurW. Gray, director of the department of physical research of the L. D. Caulk Co., entitled, Metallographic Phenomena Observed in Amalgams, read Oct. 1918, at the
Milwaukee meeting of the American Irstitute of Mining Engineers.

The splendid work therein recorded meets all the requirements of scientific investigation, equipped with facilities crvering those phases of amalgam work important to us, except adaptation; which, for twelve years, has been my special sphere of effort.

A careful consideration of this joint efiort will, I believe, lead to a recognition of the cause of our failures and to the adoption of such changes in our operative procedure as have been proved recessary.

The amalgam technic I have presented during the past five years will be found to meet all of the requirements necessary to practically uniform results in adaptation, strength, and stability; and, insofar as I am capable of the task, the procedure has been reduced to the simplest form consistant with results, and will not add more than three to five minutes time taken by the average careful amalgam operator.

For the benefit of those who may no: find the time or inclination to read the full reports of the investigations, I beg to correct some of the old time theories that have been disproved.

Exact laboratory tests have definitely shown that alloys do not deteriorate with age either before amalgamation or after completion of the filling.

Plentiful use of mercury during the $\operatorname{mix}$ is necessary to maximum strength and stability, and does not affect the amount remaining in the finished filling.

The more thoro the mix in the mortar up to the time of six minutes, the stronger and the more stable the filling; but the increase in strength after three minutes is so small that it does not justify the extra time.

The more mercury actually rubbed into the alloy the stronger the filling. The great wrong is due to our failure to remove the mercury not amalgamated.

Bulk changes, developed by time and 
apparent to the naked eye, made from rcp,resentative high grade alloys, are entirely due to excess mercury in the finished filling. Practically every well known high grade alloy contains zinc, irrespective of which, thousands of permanently good amalgam fillings have betn made. This fact clearly proves that zinc alone is not responsible for the movement.

That important attribute, adaptability to cavity walls, is lost when crepitus cievelops in amalgam, because amalgam in that condition breaks as it is compressed and these minute breaks cannot be eliminated by subsequent packing. Crepitating amalgam packs like snow, bridging the defects as snow bridges the air spaces contained, which cannot be elimirated until subjected to very heavy pressure. The more sloppy the snow, the more dense and hard the snowball can be made.

If while making the filling, the amalgam mass becomes stiff, more mercury may be added and kneaded into the mass to secure the desired plasticity without impairing the strength of the filling.

The excess mercury retained after making the mix to insure a decided plasticity, varies with the size and form of the cavity, or the time required to fil] it. This excess, commonly removed during the final kneading, is in no wise dangerous because is is always the first expressed during the packing.

The dangerous excess is the final excess that will remain in the mass after forcible squeezing during the final kneading, which will always become apparent under thoro and forcible condensation.

Smooth plasticity is not a guide to a thoro mix, smooth plasticity develops long before we reach a condition of thoro amalgamation. Vigorous mixing in the mortar for a minimum time of three minutes is the only dependable guide to a perfect mix.

It would not seem out of place to ask another question, why do dentists so commonly neglect the essential details of cavity preparation, restoration of proximal and occlusal form and contact, and perfection of finish in their amalgam work?

I would answer this question by saying that the public have been educated to believe that amalgam is undependable breause of the common discoloration. This lack of confidence has caused many to base their charge on the low cost of the material instead of the professional service. And insufficient remuneration has reduced operative skill to a very low lovel.

If this explanation is correct, it is a dcplorable situation and I trust that the presentation of a practical and successful amalgam procedure will awaken our consciences to the injustice to both operator and patient, and will lead to the adoption of a charge for real professional service, which will give to this most valuable filling material, a square deal.

\section{Discussion.}

\section{Frank J. Ryan, Chicago.}

Dr. Harper early in the paper calls your attention to our faulty procedure and in the finish he gives you the remedy, most of which you have had at your disposal six or more years. Yet the 90 per cent are still using amalgam in the old way and getting the same old results. I) . Harper with his 10 per cent or less challenges you to prove or disprove his method in open meeting, so to speak.

He tells you the importance of this work must be demonstrated in our dental scinools, otherwise the student seldom, if ever, will get an opportunity to see the results of perfect amalgam work. It has not and does not get the attention it deserves in schools and will continue to be slammed till it has a standard technic taught to the student. Too many dentists call it a cheap filling when it should be just a low priced one.

I offer no criticism of his technic at all and just wish to emphasize the decided plasticity and the removal of all 
mercury possible while packing the mix in the cavity. Use plenty of alloyenough that you can cover the margins all over at least a millimeter thick and then trim if off with an edge tool, not a burnisher. The matrix is an all important feature and is indispensable in the thcro filling of all large cavities.

\section{J. M. Barcus, Carlinville.}

I did not get a copy of Dr. Harper's paper until a day or two after I arrived hcre, and therefore cannot present a written discussion as I promised to do. But it is unnecessary to do that. A discussion of this paper in the ordinary sense cannot be made by me. It is nearly as hopeless as to discuss the Ten Commandments. They are ignored or adopted, as the case may be, but they are never discussed.

I want to congratulate the society and myself that it has a man who has given time and intelligent attention to this most important part of the practice of the dentist. I have not much doubt that 80 per cent of the fillings that dentists put in are amalgam fillings. I have not much doubt that 60 or 70 per cent of the income of the dental offices is from this same work, and I do not know of anything which can be of more importance. It is a great relief to a man of years, and who takes life seriously, to discover that an easy way is the best way. I saw Dr. Harper's clinic in 1914 amalgam fillings have been a greater joy to me than before. I have used his technic as well as my skill would allow me, and I must say generally with very much satisfaction. When I have put on the matrix, have shaped the cavity walls, have made an acute angle with the matrix, not only cutting the margins in the line of cleavage of the enamel rods, but overdoing it in the right way, leaving short rods at the bottom instead of on the outside margin, it has been a source of great relief to me to put a soft mass of amalgam up there, knowing the angles are properly filled, and then with a more rigid mass follow it up, crowding the soft mass into all the possible spaces.

Dr. Harper said that discoloration was due to a leaky filling, and that is the only thing in which I would differ with him. I think that is true, but I think it can be due also to the exudation from the dentine that is inevitable in these cases. If you put in cement linings you will thereby prevent discoloration.

Our old-time notions have been most thoroly dispelled to our very great pleasure and benefit by Dr. Harper's most capable work.

\section{J. E. Hinkins, Chicago.}

I have been very much interested in the work Dr. Harper has done and is doing in connection with amalgam, and it has been a great help to me in attaining success in the use of amalgam for the last four years.

My observations corroborate those of the assistant and have shown that amalgam fillings will save the teeth even tho the technic may be imperfect and the manipulation of the material faulty. He has pointed out to us by his experimental work that 70 to 80 per cent of these fillings leaked when inserted by the old method, and my experience is in confirmation of that declaration. So far as I know, no one has ever advanced a logical reason why amalgam saves the teeth, and if there is any member present who can enlighten us on that phase of the subject, I shall be glad to have him do so.

Dr. Harper has shown us that by his improved technic in the adaptation of amalgam, and the use of a dependable high grade alloy, from 80 to 95 per cent. of our amalgam fillings may be made sufficiently perfect in their adaptation to resist leakage at 3 to 30 pounds air pressure under practical operating conditions, with greatly increased resistance to crushing stress and flow and with comparatively uniform results.

There is no doubt that with his improved technic the amalgam mass can 
be made more decidedly plastic during the act of packing, and that with this increased plasticity a greater percentage of fillings have been made sufficiently perfect to resist air pressure of from 3 to 30 pounds.

When the packing is not done in an orderly manner, there will be lack of uniformity in the adaptation, strength and stability. I agree with the author of the paper that adaptation, resistance to crushing stress and flow and stability are one and all dependent upon the same details of operative procedure.

In regard to tooth discoloration, I have not observed this since I have been using Dr. Harper's technic, and I agree with him that this discoloration is due to oxidation, largely the result of a leaking filling. Thoro mixing for three minutes in the mortar is essential in order to bring about that degree of plasticity which is so favorable to a nice adaptation.

I was surprised to learn from the essayist that we could by adding mercury, after the filling began to crystallize, soften the material and that it makes just as good a filling. Formerly that practice was strongly condemned.

His idea of lightly tapping amalgam to enable the mass to become more homogeneous at the cervical margins is an excellent one, and we should make use of it.

The laboratory experiments he described with steel matrices are interesting, and while they are not analogous to the filling of teeth in the mouth, yet they teach us a better technic and how to manipulate the filling to greater advantage, and if we can put in a filling by his technic so that it will not leak, it is certainly a great improvement over our old methods of filling teeth.

A slow operator should not use a quick setting alloy but should use a medium setting alloy.

What he said about matrices reminded me of the old system of making them. I formerly used a knife blade with the thick part next to the gingival margin and the edge up to the cutting edge of the tooth to restore the contour, but after seeing Dr. Harper use the steel matrix with a round toothpick, it appealed to me as being much better and faster, and we can remove the excess of filling around the cervical margins much better and easier than with the old method, and this has added materially to my success in amalgam fillings.

There is no comparison between Dr. Harper's method and the way we used to ligate the matrices with strings, because they would slip and we could not keep them adjusted. It is much quicker and better if you can get at the case to the end. I have found it very important to carry out the instructions of the essayist in working out the excess of mercury around the marginal walls with the small plugger.

The four important points he enunciates in the paper are mixing in mortar for three minutes plasticity of the filling to insure adaptation, orderly packing, and to insure the most complete removal of the excess of mercury around the margins of the fillings. If this is done and as much care is devoted to it as we give to a gold filling, a porcelain inlay, or a gold inlay, I can see no reason why amalgam will not stand up far better than it is given credit for, and why we should not get better fees for a good amalgam filling than we do, and thus give amalgam a place among the filling materials where it belongs.

I have read the article by Dr. Arthur W. Gray, of Milford, Delaware, on the metallurgy of amalgam with much interest. This article was published in the Transactions of the American Institute of Mining Engineers as a supplement of Bulletin No. 144, December, 1918 . In this article the author deals with the crushing strength, dimensional changes during the hardening of amalgam, expansion and contraction, influence of temperature, influence of packing pres- 
sure, influence of handling the alloy, and so on. The author shows that alloy does not change if properly prepared and treated; that the age of the alloy does not hurt it.

I would recommend those who are interested in amalgam to procure a copy of this article and read it. I am informed that Dr. Gray's report will be published in The Journal of the $\mathrm{Na}$ tional Dental Association in the near future.

\section{General Discussion.}

Dr. Miller: I would like to have the essayist in closing the discussion say a $\mathrm{f} \in \mathrm{w}$ words on the use of pluggers in the condensation of amalgam.

Dr. H. W. McMillan, Roseville: I have attended the meetings of the Illinois State Dental Society since I became a member in 1897, about twenty-two years, with only very few exceptions, and being from a country district I have hesitated to get on the platform to discuss papers. I have used the plastic mix for years, because the dry, granular mix did not appeal to me. Because the dry mix was the method taught, I have hesitated to state my use of the plastic mix. I have used the same illustration that has been used on this platform, namely; you can pack wet snow better than you can pack chipped ice.

I remember seeing a clinic given at one of our state meetings in which the clinician had packed into a glass vial, a dry mix, as then taught, and it showed air spaces between the filling and the glass. He showed a cement lining that would fill in these air spaces. I have followed, with some modifications, the method Dr. Corbett. has shown, that of using a cement lining and packing the alloy into it to eliminate those defective spaces. I have used carbol eugenol as a paste lining underneath with good success, while also using the plastic mix.

A few years ago, when our meeting was held at Peoria, I looked for and found a three minute sand-glass timer.
You can get one for three dollars. It is a fine thing for timing the mix. This is better than holding the watch on it; you turn it over and mix the alloy until the sand has all run down, which is three minutes. I heartily recommend it as a timer.

Dr. Collins: I would like Dr. Harper to make some remarks concerning a combination of cement with amalgam, and what the advantages and disadvantages are in connection with it; whether more perfect margins can be obtained even tho the technic is not so perfect.

Dr.J.G. Reid, Chicago: I remember something like fifteen or eighteen years ago this question was brought before the Chicago Dental Society by Dr. John R. Callahan, of Cincinnati, who read a very excellent paper on amalgam. He did not at that time give the profession a detailed technic of the operation but brought to the attention of the members of the profession an important point which has been proven by this excellent paper of Dr. Harper's, namely, the imperfectness of packing amalgam into cavities were due entirely to air spaces. If you remove the air that is in an amalgam filling you have accomplished all that is to be obtained.

I recall that this paper of Dr. Callahan's was discussed by Dr. G. V. Black, and I remember how carefully at that time he tried to impress upon the audience the importance of thoroly and carefully packing amalgam, and Dr. Harper has only further by his demonstrations proven conclusively that the success of an amalgam filling depends entirely upon the care with which it is introduced into the cavity.

We look upon amalgam as a cheap flling; it is not a cheap filling at all. Dr. Pruyn, one of the old amalgam operators, spent hours and hours of time in putting in amalgam fillings. He did it most conscientiously, and he tried hard in his way to show you what was to be expected of amalgam if it was handled as 
il should be. $\mathrm{He}$ is a living monument today to that service, altho many of you regard amalgam in the light of a cheap filling. Dr. Pruyn got paid for his work, and he gave something in return for the fees he received.

Dr. Brady, St. Louis, Missouri: I did not expect to be called upon to discuss this paper, but I have done some work on dental amalgam alloys, from a metallurgical point of view, and also in working out a technic for the introduction of amalgam.

There is one point that is not clear in my mind, and I would like to ask this question: "How do you determine the correct amount of mercury to leave in the amalgam, and do you find any disadvantage if this amount is exceeded? Do you expect by manipulation, during the introduction of the filling to take care of this excess of mercury?"

I believe that if we have too great an excess of mercury we are going to cause some disturbance of the balance in our alloy. I feel there is a difference in the solubility of the various constituent metals in our alloy and we are liable to disturb the balance.

Dr. Edmund Noyes, Chicago: I do not think I can add anything of importance to what has been said in the paper and in the discussion; but there are some interesting things in regard to these matters that seem to me worih while to talk about for a few minutes, and first, to pursue just a step further what has just been said about disturbing the balance of the alloy. That relates to the question of how much, besides mercury, is squeezed out in the packing of these fillings, and what proportions there are of and dissolved metals which go out with an excess of mercury. This would seem to be one further question for passible investigation, if there are any metals in solution in the mercury squeezed out, it would likely promote expansion in the finished filling rather than contraction, because the more readily soluble tin is likely to come out in greater amount than the less soluble silver, and increasing the relative proportion of silver would tend to expansion if it was a perfectly balanced alloy to begin with.

I was very glad to hear the tribute which Dr. Reid paid to Dr. Pruyn in regard to his amalgam work and what he has said and done on that question. It was well deserved and I am not quite familiar enough with what has been done and published to speak with so much confidence as I would like to, but it seems to me, as I have listened to this paper, that we have probably arrived at a standard technic for the packing of amalgam. Between 1890 and 1900 Dr. Black carried on an elaborate series of experiments in regard to amalgam alloys. $\mathrm{He}$ wholly revolutionized the opinions and the knowledge of the profession in regard to them, and standardized the making of amalgam alloys with reference to expansion or contraction, and since that time there has been no change or improvement in the scientific basis of amalgam alloy construction. It seems to me, Dr. Harper has rendered a similar service in regard to the manipulative details in the handling of amalgam. It is as important as the first service. The possession of a well balanced alloy that will neither shrink nor expand is not enough to enable us to make good amalgam work but Dr. Harper has developed a technic which must be used if we wish to do what amalgam can be made to accomplish. Without for a moment disparaging in any way the great amount of work that has been done by numerous other men, it seems to me probable that Dr. Harper's work will settle this question for a good while in the future, just as the question of expansion and contraction was settled by Dr. Black; just as the question of cavity preparation was scientifically enunciated and standardized by him, so this work of Dr. Harper's, it seems to me, will standardize the packing of amalgam. 
Dr. G. H. Henderson, Chicago: The Illinois State Dental Society has done more than any other society in my judgment to introduce the proper technic of amalgam, and those of us who have been attending these meetings for years will remember the very many discussions we have had on this subject. I want to say to you, that up to a few years ago I had put in many hundreds of amalgam fillings, but I did not know how to make amalgam fillings until Dr. Harper showed me. He has given us a technic that, if properly carried out, will enable us to put in amalgam fillings properly. It is all right to have a very plastic mix of amalgam before you put it into a cavity, but after you get it packed in a cavity you have no business in having a surplus of mercury, and that is where a great many make a mistake.

Dr. Fred C. Noyes, Jacksonville: I want to thank and compliment the essayist on that part of the paper which I heard; I did not hear the first part of it. I have been a friend of amalgam ever since I went into the practice of dentistry, and $I$ received my instruction under Dr. A. O. Kulp, of the Iowa State University, in the eighties.

Dr. Kulp's instruction was to use a soft mix of amalgam, and those fillings we inserted with a tamping and burnishing movement and each time a little pellet of absorption cotton was used to brush away the surplus mercury, as we proceeded.

My gold fillings have not lasted as long in some cases as the amalgam fillings, altho I have used the same care as in putting in an alloy filling. I have been a staunch friend of amalgam all my professional life.

Dr. C. B. Mead, Rockford: It has amused me in a way to hear some of our men talk of the fine amalgam fillings they put in ten or fifteen or twenty years ago, when practically every one looked like some of the worst pictures Dr. Harper showed on the screen.

The other day I extracted a tooth from an old man, an upper molar, that had in the occlusal surface a fair sized amalgam filling that had been there for thirty-five years. I was interested in knowing the condition of that filling. I ground the tooth at its long axis and exposed half of the filling to the pulp chamber. There had been some decay and it had shrunken so that there was a space around the filling. The discolored dentine had progressed near to the pulp chamber. All this leathery decay around the filling and this discolored dentine was black, with the pulp alive. That brings to my mind the possible connection between our silver reduction method of treating root-canals and the discoloration of that dentine.

Nearly all of you saw the clinic given by Dr. Fuqua yesterday, and you noticed the tooth he showed, how the silver oxid had progressed far thru the dentinal tubuli and discolored the whole root, thereby sterilizing it. It is evident to me that some sort of action took place on the silver in that amalgam filling that produced some oxid that progressed down thru and sterilized the dentine and stopped further decay, and not being a severe irritant it kept the pulp alive.

I do not believe we can differ with Dr. Harper. I have been using this method ever since he brought it out five or six years ago with very gratifying results. The main thing to my mind is, how can we get the four hundred men not here today to use it?

Dr. Harper (Closing): The question was asked regarding the variety in size and form and the best kind of pluggers for amalgam work.

After having used a very extended array of pluggers, my work during the past year or two has been done with four smooth faced pluggers (the serrations having been ground off with a coarse carborundum wheel in the dental 
engine). Following are the size and forms:

Large round flat faced plugger 35 tenths of a millimeter in diameter.

Medium round flat faced plugger 25 tenths of a millimeter in diameter.

Parallelogram flat faced plugger 25 by 14 tenths of a mm. in diameter.

Parallelogram flat faced plugger 18 by 12 tenths of a mm. in diameter.

The large and medium pluggers are used for the general building of the filling; the smaller parallelograms are used to reach well into irregularities and angles, and for stepping the cavity walls.

The use of flat smooth faced pluggers has proved very satisfactory and has been found to meet every requirement in my amalgam work, and the plastic amalgam will not adhere to the smooth face during the packing.

Building amalgam fillings by the ust of burnishers (as suggested by the late Dr. Callahan and by Dr. C. P. Pruyn) will greatly improve the naked eye appearance of our fillings, but does not improve the adaptation when the fillings are tested by air pressure, probably because this amalgam procedure does not provide sufficient compression of the amalgam against the cavity walls to resist air pressure.

The amalgam technic provides for all these difficulties.

Test fillings made for me by representative operators, using amalgam in combination with cement, have proved to be undependable in their adaptation; and fillings so made are very weak under crushing stress, under which circumstances I would condemn this technic as devoid of merit.

It will be found safer to use amalgam extra plastic rather than risk the development of any degree of creaking during the filling of the first two third of average medium and large cavities. A thoro amalgam technic will always insure the complete removal of the un- amalgamated mercury, and the finished filling will set as quickly and as hard, and will contain as small a per cent of mercury as would be the case if a large portion of the excess mercury had been expressed during the final kneading.

The removal of excess mercury does not carry with it a sufficient amount of the more soluble metals to disturb in the slightest degree any of the essential qualities in our amalgam fillings as far as can be detected with our present day testing apparatus.

I would consider it inadvisable to use expressed mercury (except on the one filling for which it was made) without refining, because it would require but a short time to develop a mixture unknown in the quantity and character of its contents and the low price of mercury does not justify such economy.

It is my experience to observe that those high grade alloys that require a large per cent of mercury to insure thoro amalgamation (mercury eight to nine parts, alloy five, by weight) give up less mercury during the mixing and packing, and notwithstanding this large mercury content such amalgams will be found to make the strongest fillings (a statement contrary to general belief). And such an amalgam filling costs the dentist much less as much less alloy is required to make the filling.

The question of strength and stability in our amalgam fillings is not governed by the per cent of mercury in the finished filling, the danger lies in our failure to remove that mercury not amalgamated, by a thoro, forceful and orderly condensation, the operator cannot guard against this danger by using less murcery for the mix, or by trying to express the excoss during the final mixing or kneading because this excess mercury only become's apparent under thoro manipulation and packing, the one opportunity to remove it completely, by the use of a proper technic at the proper time. 